\title{
Profiling the BLADE-ON-PETIOLE gene expression in the abscission zone of generative organs in Lupinus luteus
}

\author{
Kamil Frankowski ${ }^{1}$ Emilia Wilmowicz ${ }^{1,3}$ - Agata Kućko ${ }^{1,3}$ - Agnieszka Zienkiewicz ${ }^{1,4}$ • \\ Krzysztof Zienkiewicz ${ }^{2,4} \cdot$ Jan Kopcewicz $^{1}$
}

Received: 26 May 2015/Revised: 3 August 2015/Accepted: 22 September 2015/Published online: 6 October 2015

(C) The Author(s) 2015. This article is published with open access at Springerlink.com

\begin{abstract}
The great agronomic potential of Lupinus luteus, species widely cultivated in many European countries as well as Australia, is strongly affected by premature and excessive generative organ abscission, mainly flowers. The unwanted process takes place in a specialized group of cells, called abscission zone (AZ). During their development they become competent to respond to external and internal factors, including phytohormones. Recently it was shown that the formation of AZ cells in Arabidopsis thaliana is coordinated by transcription factors, BLADEON-PETIOLE (BOPs). There is no such data, excluding tobacco plants, about BOP-dependent regulation of organ abscission in crop plants. In this work, we examined LIBOP mRNA content during generative organs AZ development and functioning. The high accumulation of LIBOP transcript was accompanied by the differentiation of morphologically distinct cells at the base of the mature flower pedicel. Moreover, enhanced $L l B O P$ expression was
\end{abstract}

Communicated by Y. Wang.

Emilia Wilmowicz

emwil@umk.pl

1 Chair of Plant Physiology and Biotechnology, Nicolaus Copernicus University, 1 Lwowska Street, 87-100 Toruń, Poland

2 Department of Cell Biology, Nicolaus Copernicus University, 1 Lwowska Street, 87-100 Toruń, Poland

3 Centre for Modern Interdisciplinary Technologies, Nicolaus Copernicus University, 4 Wileńska Street, 87-100 Toruń, Poland

4 Department of Biochemistry, Cellular and Molecular Biology of Plants, Estación Experimental del Zaidín, Consejo Superior de Investigaciones Científicas (CSIC), Profesor Albareda 1, 18008 Granada, Spain observed in the active AZ, and was regulated by factors, which can strongly affect generative organ abscission. All these data indicate that $L I B O P$ is involved in the abscission zone formation and functioning in $L$. luteus.

Keywords BOP · Organ abscission · Phytohormones · Yellow lupin

\section{Introduction}

Excessive abscission of generative organs, particularly Lupinus luteus flowers, represents important economical drawbacks for cultivators (Frankowski et al. 2014; Prusiński and Borowska 2007; van Steveninck 1958). Abscission zone (AZ) of generative organs occurs in the specialized group of cells at a predetermined site on their detachment. These cells are small, cytoplasmically dense, isodiametric and morphologically distinct from others (Estornell et al. 2013). AZ during plant growth becomes competent to respond to specified signals, thus initiating separation events and consequently leading to breakdown in cell adhesion. Various transcription factors, enzymes and phytohormones have been shown to regulate AZ formation and development. It is widely accepted that exogenous ethylene (ET) and abscisic acid (ABA) are positive regulators of abscission, whereas auxin (IAA) can act as an inhibitor of that process (Estornell et al. 2013; Taylor and Whitelaw 2001). Critical for differentiation of corolla AZ in Arabidopsis thaliana are BLADE-ONEPETIOLE1 and 2 (BOP1, BOP2) transcription factors, which belong to the NON-EXPRESSOR OF PR1 (NPR1) protein family-responsible for the regulation of systemic acquired resistance (SAR) and salicylic acid-induced broad-spectrum defense against pathogens (Ha et al. 2003, 
2004; Rochon et al. 2006). BOPs contain conserved structural motifs which commonly occur in NPR1-like proteins. Loss-of-function bop1 bop2 in A. thaliana mutant are defective in floral organ abscission (Ha et al. 2003; Norberg et al. 2005). Interestingly, recent works have indicated that BOPs coordinate many other plant growth and developmental processes: embryogenesis, meristem determinacy, leaf patterning, inflorescence architecture as well as flower development (Khan et al. 2014).

In our previous work we have identified for the first time the homologue of BOP gene in Lupinus luteus and we have shown that $L I B O P$ is involved in root nodule development and functioning (Frankowski et al. 2015). At this point, it would be important to shed light on the regulatory role of $\angle I B O P$ in the generative organ abscission, which is another yield determining process that can affect crop growth. Therefore, in this paper the LIBOP expression pattern was characterized during generative organ $\mathrm{AZ}$ formation and development. To verify if the $L I B O P$ cDNA content is correlated with changes in the cell structure, we described AZ anatomy. Considering ET and ABA as crucial phytohormones inducing abscission, we also measured LlBOP expression after their application.

\section{Materials and methods}

\section{Plant material}

The epigonal cultivar Taper of yellow lupine (Lupinus luteus) was used in the study. The L. luteus seeds (Poznan Plant Breeding Tulce, Wiatrowo, Poland) were treated with Sarfun (250 $\mathrm{cm}^{3} 100 \mathrm{~kg}^{-1}$ seeds) (Organika-Sarzyna S.A., Nowa Sarzyna, Poland), inoculated with Bradyrhizobium lupini (Nitragina $3 \mathrm{~g} / \mathrm{kg}$ seeds, "BIOFOOD S.C", Wałcz, Poland) for $2 \mathrm{~h}$ and subsequently sown in $11 \mathrm{dm}^{3}$ pots (5 seeds per pot, with a spacing of $0.02 \mathrm{~m}$ ) filled with class $\mathrm{V}$ soil material. The seeds were planted at a depth of $0.03-0.04 \mathrm{~m}$. The lupine was grown in a cultivated chamber at a temperature of $22 \pm 1{ }^{\circ} \mathrm{C}$ under long day conditions $\left(110 \mu \mathrm{mol} \mathrm{m} \mathrm{s}^{-2}\right.$, cool white fluorescent tubes by Polam, Warsaw, Poland).

The pedicels fragments containing abscission zones (1 $\mathrm{mm}$ above AZ-distal side and $1 \mathrm{~mm}$ below AZproximal side) were collected at the following developmental stages: 1st, flower bud; 2nd, 5-mm-long flower bud; 3rd, 8-mm-long flower bud with visible yellow petals, 4th, 10-mm-long flower bud; 5th, 12-mm-long unopened flower; 6th, 14-mm-long opened flower; 7th, a pollinated flower; 8th, 6-mm-long pod in the flower; 9th, 10-mm-long pod with senescent petals; 10th, 12-mm-long pod; 11th, 14-mm-long pod; 12th, 20-mm-long pod; 13th, 30-mmlong pod (Fig. 1a).
4Fig. 1 a Stages of generative organ development in Lupinus luteus: flowers (1-7), pods (8-13). A small pod set in a flower from stage 8 is shown above (for details see "Materials and methods"). Bars $1 \mathrm{~cm}$. b The expression level of $L I B O P$ (related to $L I A C T$ ) in the developing pedicels of flowers and pods. Material was harvested from 13 stages of generative organ development (For details see "Materials and methods" section). The expression activity was measured in three independent biological replicates. $\mathrm{SE} \pm$ is marked on the bars. c Toluidine blue staining of L. luteus floral abscission zone sections. b-h Light microscopy sections of abscission zone cells from flowers at different stages of development (small rectangles). i Enlarged, fully developed AZ cells. $A Z$ abscission zone; $F$ flower; $P$ flower pedicel; $S$ stem. Bars $2 \mathrm{~mm}$ in (a-h); $700 \mu \mathrm{m}$ in (i)

Furthermore, we investigated the role of $L I B O P$ in the activation of abscission processes. For this purpose, the AZ-containing tissues were collected at three different variants: (1) fully opened flowers with green pedicels from sixth stage of flower development (inactive abscission zone, IN AZ, control), that showed no signs of abscising; (2) flowers with symptoms of senescence, yellow and dehydrated pedicels (active abscission zone, A AZ). Additionally, individual flowers were removed from plants by razor blade above the $\mathrm{AZ}$, which turned the abscission process, and pedicels were excised from the plant after $24 \mathrm{~h}$; and (3) variant-activated abscission zone, AC AZ.

To evaluate the role of phytohormones in the regulation of $L I B O P$ expression and indirectly in AZ functioning we performed several treatments. Pedicels containing IN AZ were treated with $0.1 \mathrm{mM} \mathrm{ABA} ; 0.01 \mathrm{mM}$ NDGA (inhibitor of ABA biosynthesis); $100 \mu \mathrm{l}^{-1} \mathrm{ET}$; $0.1 \mathrm{mM} \mathrm{ACC}$ (ET precursor); $0.1 \mathrm{mM} \mathrm{AVG} \mathrm{and} 100 \mu \mathrm{l}^{-1} \mathrm{NBD}$ (inhibitors of ET synthesis and action, respectively). ET or NBD were put via a syringe into the glass containers, which were placed on pots with enclosed plants. Other phytohormone solutions were prepared in $0.1 \%$ Tween 20 and were applied by small brushes directly onto the AZ. The material was collected after 2, 4, 6, 8, 16 and $24 \mathrm{~h}$ of treatment.

Plant material for all expression analyses was frozen in liquid nitrogen and stored at $-80{ }^{\circ} \mathrm{C}$ until RNA isolation procedures. All experiments were designed in three independent biological replication

\section{Quantitative RT-PCR analyses of the $\mathrm{LlBOP}$ expression}

The gene expression analyses were performed by RealTime PCR (RT-qPCR) with a LightCycler 2.0 CarouselBased System (ROCHE Diagnostics GmbH, Germany) and the LightCycler TaqMan Master Kit (ROCHE Diagnostics GmbH, Germany) with using specific primers and probes (Table 1). All expression procedures were performed according to Frankowski et al. (2015). 
A

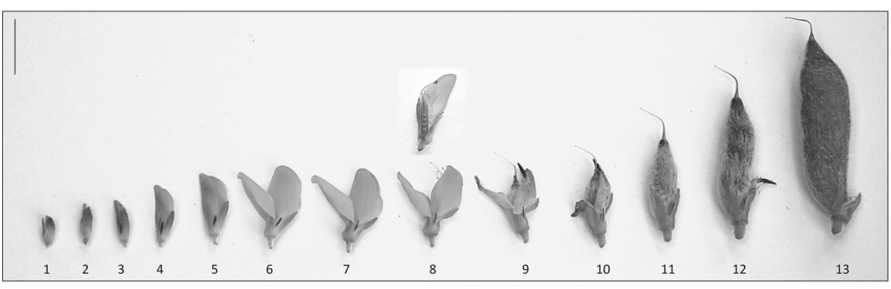

C
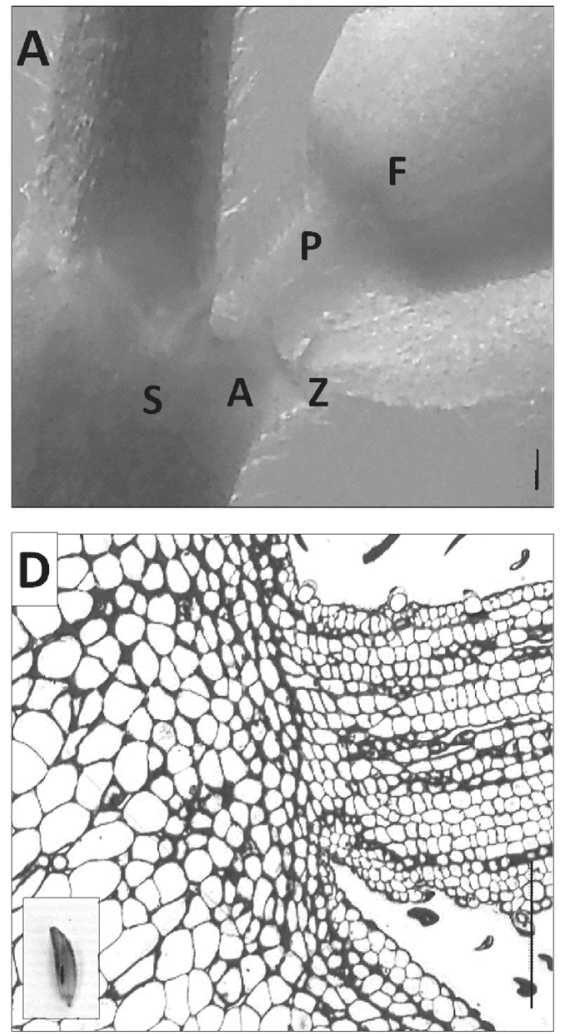

G

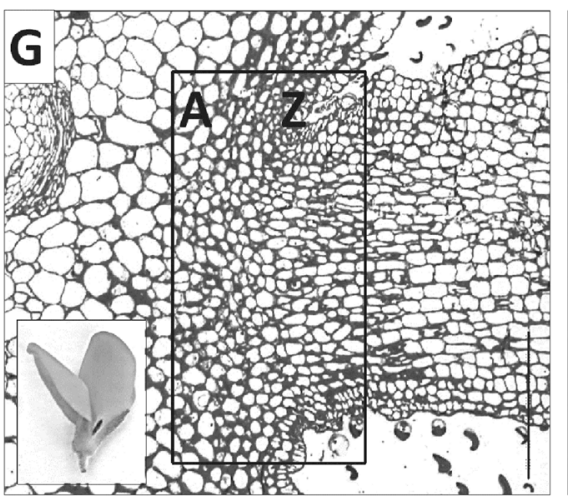

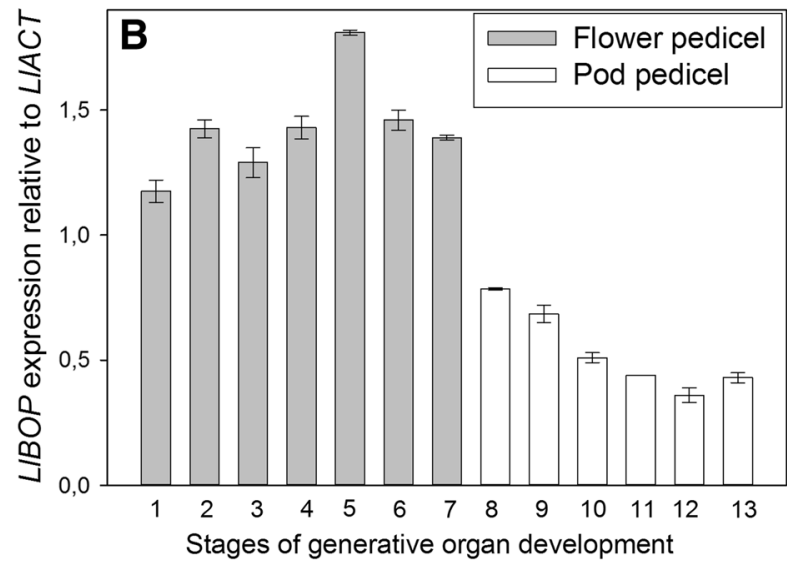
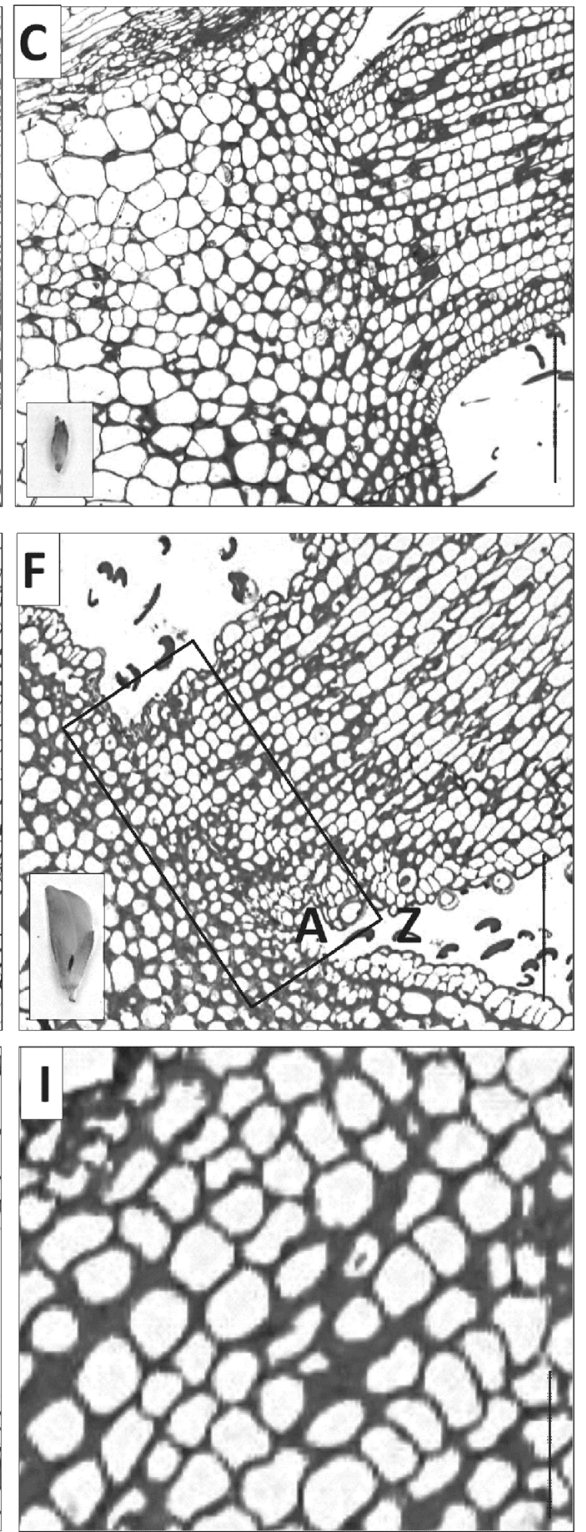
Relative quantification was performed using standard curves from serial dilutions of cDNA. The efficiency tested was $>99 \%$. The computer application used for the analyses was LightCycler Real-Time PCR Systems (ROCHE Diagnostics $\mathrm{GmbH}$, Germany), while for the calculations and graphs MS Office Excel (Microsoft) and SigmaPlot 2001 v.7.0, respectively, were used. qPCR reactions were carried out in triplicate for each RNA sample. All data are the results of three separate samples with two replications of each and presented as mean \pm standard error (SE).

\section{Microscopy sample preparation and histological studies}

Flower pedicels used for microscopic analyses were fixed with $4 \%$ paraformaldehyde and $0.2 \%$ glutaraldehyde in

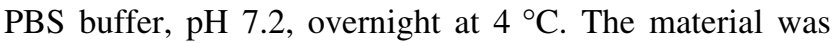
dehydrated in increasing ethanol series including $10 \mathrm{mM}$ dithiothreitol: 30, 50, 70, 90 and $100 \%$ (v/v) concentrations, supersaturated and then embedded in BMM resin (butyl methacrylate, methyl methacrylate, $0.5 \%$ benzoin ethyl ether, $10 \mathrm{mM}$ dithiothreitol; Fluka, Buchs, Switzerland). Semithin sections $(1 \mu \mathrm{m})$ were cut on an Ultracut microtome (Reichert-Jung, Germany) and were placed on glass slides covered with Biobond (British Biocell International, Cardiff, UK). For general histological observations, sections were stained with $0.05 \%$ toluidine blue. The samples were observed in a LM Zeiss Axioplan microscope (Carl Zeiss, Germany), whereas the images were obtained with a ProGres C3 digital camera using the ProGres CapturePro 2.6 software (Jenoptik AG, Germany).

\section{Results and discussion}

A novel transcriptional factor encoding for $B L A D E-O N$ PETIOLE (BOP) gene was previously shown to be involved in many aspects of plant development, e.g. leaf morphogenesis, meristematic activity and organ abscission (Couzigou et al. 2012; Ha et al. 2003, 2004; Hepworth et al. 2005; Khan et al. 2014). In our latest work, we demonstrated the significant role of $L I B O P$ in the control of nodulation and nitrogen-fixing bacteroids functioning in Lupinus luteus (Frankowski et al. 2015). From the agronomic point of view another critical factor for crop plants is the formation and successful development of flowers and pods with seeds afterwards. In L. luteus most of the flowers are detached and this phenomenon has a strong effect on yielding (Frankowski et al. 2014). In order to determine the stage of development in which generative organs $\mathrm{AZ}$ is formed, we studied the cellular changes and expression of LIBOP (potential coordinator of that process) in the pedicels.

Our studies showed that LIBOP cDNA accumulation during flower pedicel development was significantly higher in comparison to pods pedicles (Fig. 1b). The level of LIBOP mRNA kept increasing in the pedicels AZ until stage 5, and then decreased gradually. A structural analysis using microscopy techniques revealed that the abscission zone is located at the base of the flower pedicel (Fig. 1c) and the formation of morphologically different cells within the AZ started from stage 5 of flower development [Fig. 1c $(\mathrm{f}-\mathrm{h})]$. That cells are smaller, densely packed and round, distinct from the adjacent cells below the AZ (proximal side: nearest tissue to the plant body) and above the AZ (distal side: belongs to the flower) [Fig. 1c(i)]. On the basis of our results, we suggest that $L I B O P$ is involved in $\mathrm{AZ}$ differentiation in L. luteus. Similar observations were obtained for A. thaliana and Nicotiana tabacum (Couzigou et al. 2012; Khan et al. 2012; McKim et al. 2008; Wu et al. 2012). In A. thaliana, BOP $1 / B O P 2$ were expressed in the $A Z$ prior to other abscission-related gene markers and their cDNAs content was maintained throughout the development (Khan et al. 2012; McKim et al. 2008). AtBOP2 was strongly accumulated at the base of the floral organs during late stages of their development in the area of hypothetical place of flower abortion. In turn, in Nicotiana tabacum, $N t B O P$ restricted growth was observed in the differentiating corolla abscission zone by inhibiting longitudinal cell expansion (Wu et al. 2012).

To investigate the involvement of $L I B O P$ in subsequent AZ functioning, we measured its expression in activated

Table 1 Specific primers and probes used in the RT-qPCR reactions

\begin{tabular}{|c|c|c|c|c|}
\hline Name of gene & GeneBank accession no. & Primer sequence $5^{\prime}-3^{\prime}$ & UPL probe no. & Product size (bp) \\
\hline$\angle I B O P$ & KC792647.1 & $\begin{array}{l}\text { qRT-PCR } \\
\text { F: GGAACTCGTCAAGCTCATGG } \\
\text { R: GGCGTAGTGTAAGGCCAATG }\end{array}$ & 133 & 77 \\
\hline LIACT & KP257588 & $\begin{array}{l}\text { qRT-PCR } \\
\text { F: TGGACGTACTACAGGTATTGTGC } \\
\text { R: ATGGGCACTGTATGGCTCAC }\end{array}$ & 9 & 60 \\
\hline
\end{tabular}

$F$ forward primer, $R$ reverse primer 
and active AZ. The $L I B O P$ expression was almost three times higher in the pedicels with activated AZ in comparison to inactive AZ (Fig. 2a). Moreover, increased level of $L l B O P$ transcript was also observed in senescent pedicels with visible AZ. Therefore, we speculated that other factors regulating $\mathrm{AZ}$ activation may affect $L l B O P$ expression. AZ functioning is highly dependent on phytohormone actions and we put forward a hypothesis that $L I B O P$ cDNA accumulation also is regulated by major activators of organ separation-ET and ABA (Agusti et al. 2009; Aneja et al. 1999; Taylor and Whitelaw 2001). Stimulating effect of ET and ABA on LlBOP transcriptional activity was observed as early as $2 \mathrm{~h}$ after the hormones application, but in case of ET the effect was almost four times as higher as in inactive $\mathrm{AZ}$ and twice higher than in the case of ABA (Fig. 2b, c). In both cases high mRNA
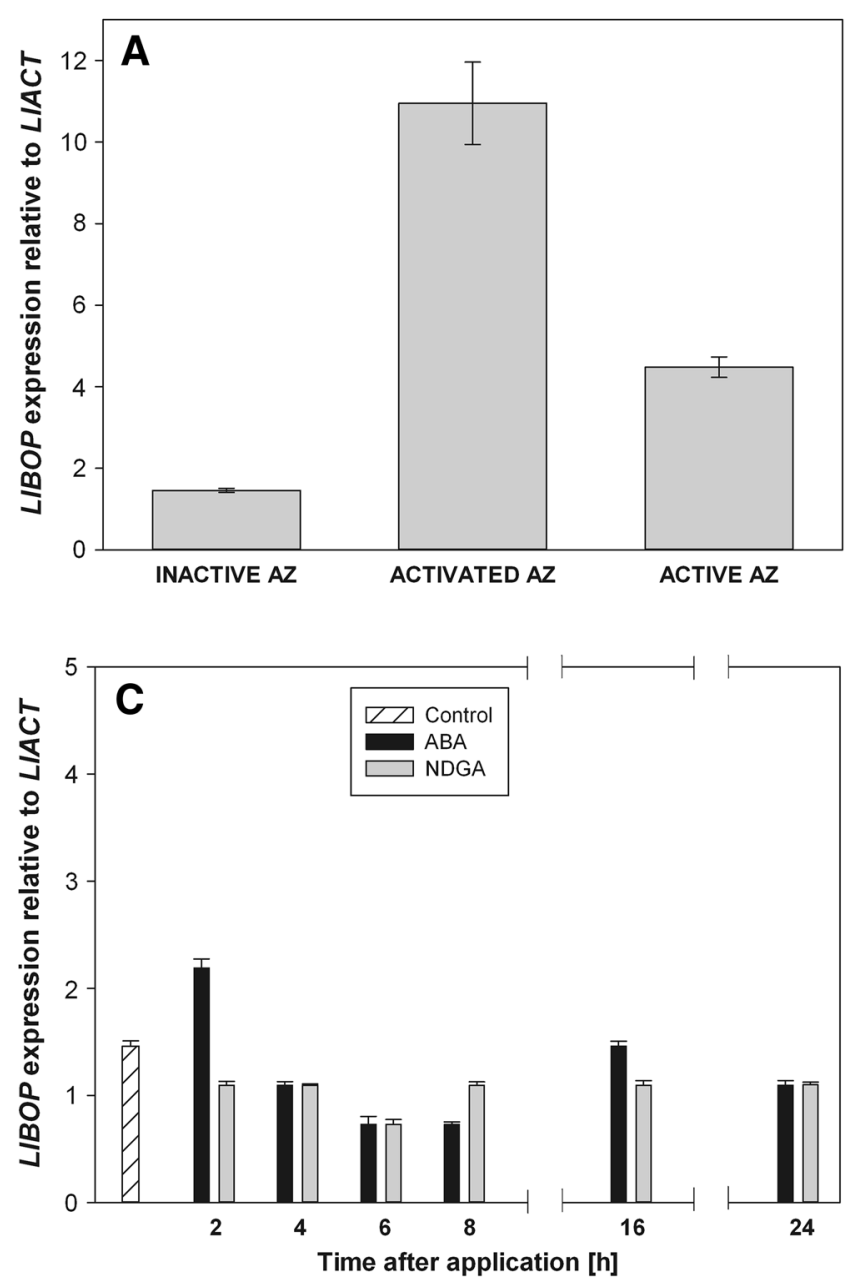

Fig. 2 LIBOP expression pattern (related to LlACT) in L. luteus floral pedicels. a Inactive AZ, from fully opened flowers (stage 5), treated with $0.1 \%$ Tween 20 , was the control. AZ was activated by removing of a flower (Activated AZ), Tissues containing Active AZ were harvested from naturally senescent flowers with yellow pedicels. RTqPCR analysis of LIBOP mRNA in flower pedicels under treatments content was extremely decreased and reached the similar value $(16 \mathrm{~h})$ as control AZ (Fig. $2 \mathrm{a}-\mathrm{c})$. It may be connected with turning on the ET and $\mathrm{ABA}$ actions on certain process, in this case activation of abscission. In turn, application of biosynthesis or action inhibitors of those hormones (NDGA, NBD or AVG) had no effect on LlBOP transcriptional activity (Fig. 2b-d). Blockage of ET or ABA biosynthesis/action strongly supports the hypothesis about their essential stimulating role in $\angle I B O P$-regulated AZ functioning. Additionally, low $L I B O P$ expression after ACC treatment may be a result of the fact that ET can influence on its own biosynthesis at the level of ACC oxidase gene (Fig. 2d). Thus, application of a precursor can be insufficient to hormone production and inducing physiological reaction. Additionally, it confirms that ET, not $\mathrm{ACC}$, is a signal molecule in the examined process. On the
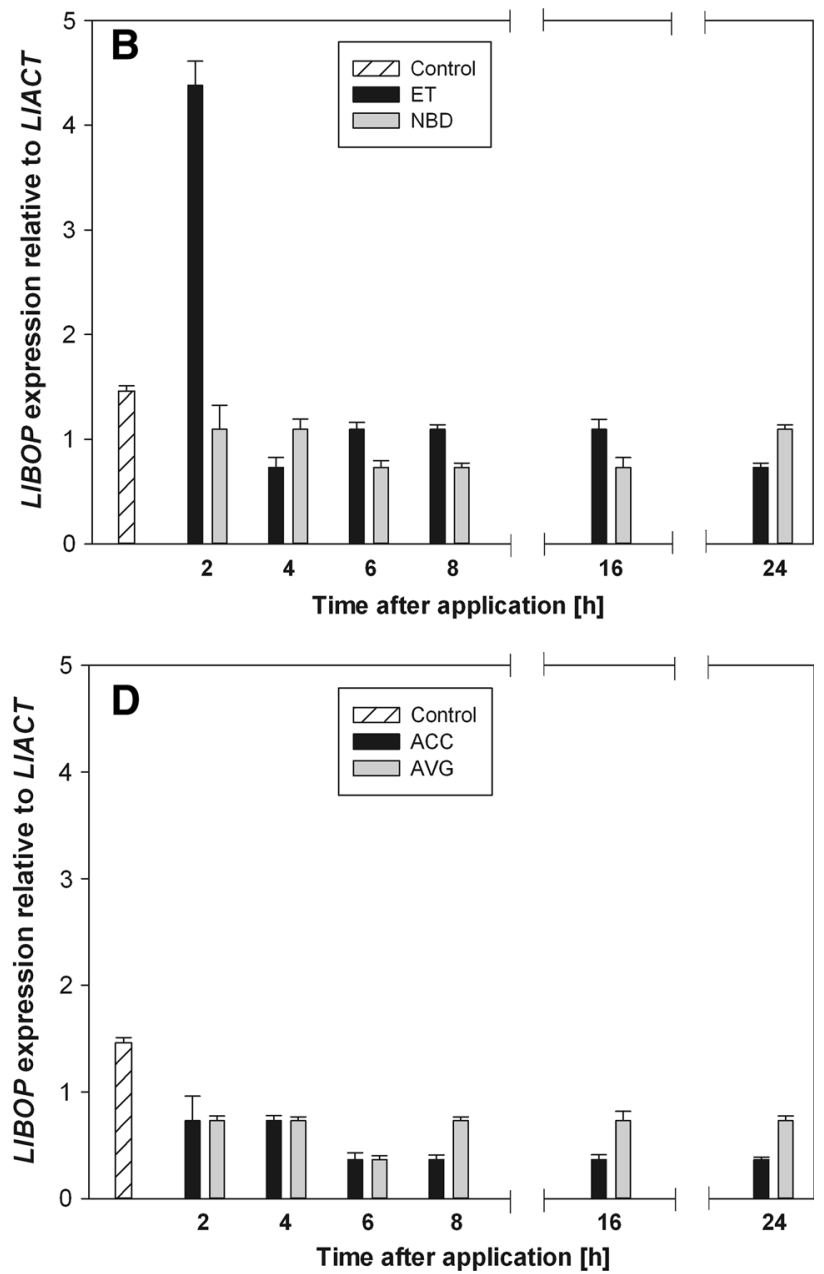

with (b) ethylene, ET or 2,5-norbornadiene, NBD; c abscisic acid, ABA or nordihydroguaiaretic acid, NDGA; d 1-aminocyclopropane1-carboxylic acid, ACC or aminoethoxyvinylglycine, AVG. AZ abscission zone. The expression activity was measured in three independent biological replicates. $\mathrm{SE} \pm$ is marked on the bars 
basis of all the results obtained here, it is suggested that $\angle I B O P$ is involved in both $\mathrm{AZ}$ formation and functioning through phytohormone action (ET and ABA). In A. thaliana NPR1 proteins regulate the expression of PR (PATHOGENESIS-RELATED) genes (Maier et al. 2011). Moreover, ethylene-induced AZ activation in Sumbucus nigra leaflet strongly increased the mRNA level of PR genes (Coupe et al. 1997). Thus, it cannot be completely excluded that changes in $L I B O P$ expression induced by exogenous phytohormones could be, in a certain degree, connected with a stress, which is one of the factors stimulating conversion leading to organ abortion.

In view of the disadvantageous phenomenon of premature and excessive generative organ abscission in lupine, it is of extreme importance to get the knowledge about the mechanisms regulating these processes. Our work confirms that $L l B O P$, examined here at the transcriptional level, is a significant component of generative organ abscission mechanisms, which is an important process that is directly translated into yields. It also provides informative suggestions for future manipulation of the events to achieve a controllable abscission, not only in lupine, but also in other crop plants. However, for a more precise explanation of this phenomenon, in the nearest future we would like to study the localization of $L I B O P$ transcript during AZ formation and under abscission stimulators or inhibitors treatments.

Author contribution statement Kamil Frankowski and Emilia Wilmowicz designed and carried out the experiments, analyzed the data and wrote the manuscript. Agata Kućko carried out the experiments and prepared the figures and charts. Agnieszka Zienkiewicz, Krzysztof Zienkiewicz and Agata Kućko conducted the microscopy experiments. Jan Kopcewicz helped in the manuscript preparation.

Acknowledgments This work has been funded by Polish Ministry of Agriculture and Rural Development grant no 149/2011. A. Kućko thanks the eidA3-ceiA3 consortium for funding throughout the program for Ph.D. co-supervision for foreign students.

Open Access This article is distributed under the terms of the Creative Commons Attribution 4.0 International License (http://crea tivecommons.org/licenses/by/4.0/), which permits unrestricted use, distribution, and reproduction in any medium, provided you give appropriate credit to the original author(s) and the source, provide a link to the Creative Commons license, and indicate if changes were made.

\section{References}

Agusti J, Merelo P, Cercos M, Tadeo FR, Talon M (2009) Comparative transcriptional survey between laser-microdissected cells from laminar abscission zone and petiolar cortical tissue during ethylene-promoted abscission in citrus leaves. BMC Plant Biol 9:127. doi:10.1186/1471-2229-9-127

Aneja M, Gianfagna T, Ng E (1999) The roles of abscisic acid and ethylene in the abscission and senescence of cocoa flowers. Plant Growth Regul 27:149-155

Coupe SA, Taylor JE, Roberts JA (1997) Temporal and spatial expression of mRNAs encoding pathogenesis-related proteins during ethylene-promoted leaflet abscission in Sambucus nigra. Plant Cell Environ 20:1517-1524

Couzigou JM, Zhukov V, Mondy S, Abu el Heba G, Cosson V, Ellis TH, Ambrose M, Wen J, Tadege M, Tikhonovich I, Mysore KS, Putterill J, Hofer J, Borisov AY, Ratet P (2012) NODULE ROOT and COCHLEATA maintain nodule development and are legume orthologs of Arabidopsis BLADE-ON-PETIOLE genes. Plant Cell 24:4498-4510

Estornell LH, Agustí J, Merelo P, Talón M, Tadeo FR (2013) Elucidating mechanisms underlying organ abscission. Plant Sci 199-200:48-60

Frankowski K, Wilmowicz E, Kućko A, Mączkowski R, Marciniak K, Kopcewicz J (2014) The generative development of traditional and self-completing (restricted branching) cultivars of white lupin (Lupinus albus L.), yellow lupin (L. luteus L.) and narrowlafed lupin (L. angustifolius L.) grown under different phytotron conditions. Plant Breed Seed Sci 69:47-57

Frankowski K, Wilmowicz E, Kućko A, Zienkiewicz A, Zienkiewicz $\mathrm{K}$, Kopcewicz J (2015) Molecular cloning of BLADE-ONPETIOLE gene and expression analyses during nodule development in Lupinus luteus. J Plant Physiol 179:35-39

Ha CM, Kim GT, Kim BC, Jun JH, Soh MS, Ueno Y, Machida Y, Tsukaya H, Nam HG (2003) The BLADE-ON-PETIOLE 1 gene controls leaf pattern formation through the modulation of meristematic activity in Arabidopsis. Development 130:161-172

Ha CM, Jun JH, Nam HG, Fletcher JC (2004) BLADE-ONPETIOLE1 encodes a $\mathrm{BTB} / \mathrm{POZ}$ domain protein required for leaf morphogenesis in Arabidopsis thaliana. Plant Cell Physiol 45:1361-1370

Hepworth SR, Zhang Y, McKim S, Li X, Haughn GW (2005) BLADE-ON-PETIOLE-dependent signaling controls leaf and floral patterning in Arabidopsis. Plant Cell 17:1434-1448

Khan M, Xu M, Murmu J, Tabb P, Liu Y, Storey K, McKim SM, Douglas CJ, Hepworth SR (2012) Antagonistic interaction of BLADE-ON-PETIOLE1 and 2 with BREVIPEDICELLUS and PENNYWISE regulates Arabidopsis inflorescence architecture. Plant Physiol 158:946-960

Khan M, Xu H, Hepworth SR (2014) BLADE-ON-PETIOLE genes: setting boundaries in development and defense. Plant Sci 215-216:157-171

Maier F, Zwicker S, Hückelhoven A, Meissner M, Funk J, Pfitzner AJ, Pfitzner UM (2011) NONEXPRESSOR OF PATHOGENESIS-RELATED PROTEINS1 (NPR1) and some NPR1-related proteins are sensitive to salicylic acid. Mol Plant Pathol 12:73-91

McKim SM, Stenvik GE, Butenko MA, Kristiansen W, Cho SK, Hepworth SR, Aalen RB, Haughn GW (2008) The BLADE-ONPETIOLE genes are essential for abscission zone formation in Arabidopsis. Development 135:1537-1546

Norberg M, Holmlund M, Nilsson O (2005) The BLADE ON PETIOLE genes act redundantly to control the growth and development of lateral organs. Development 132:2203-2213

Prusiński J, Borowska M (2007) Degree of success of legume cultivars registered by the center for cultivar testing over the period of market economy. Acta Sci Pol Agric 6:3-16

Rochon A, Boyle P, Wignes T, Fobert PR, Despres C (2006) The coactivator function of Arabidopsis NPR1 requires the core of its $\mathrm{BTB} / \mathrm{POZ}$ domain and the oxidation of $\mathrm{C}$-terminal cysteines. Plant Cell 18:3670-3685 
Taylor JE, Whitelaw CA (2001) Signals in abscission. New Phytol 151:323-339

van Steveninck RFM (1958) Factors affecting the abscission of reproductive organs in yellow lupins (Lupinus luteus L.). I. The effect of different patterns of flower removal. J Exp Bot $8: 373-381$
Wu XM, Yu Y, Han LB, Li CL, Wang HY, Zhong NQ, Yao Y, Xia GX (2012) The tobacco BLADE-ON-PETIOLE2 gene mediates differentiation of the corolla abscission zone by controlling longitudinal cell expansion. Plant Physiol 159:835-850 